\title{
IMS Learning Design, evoluindo de Objetos de Aprendizagem para Atividades de Aprendizagem
}

\author{
Renato Luís de Souza Dutra* \\ Liane Margarida Rockenback Tarouco** \\ Mary Lucia Pedroso Konrath ${ }^{* * *}$
}

Resumo. Com a crescente utilização da tecnologia de objetos de aprendizagem, surgiram diversas especificações para normatizar o desenvolvimento destes módulos. A criação destas especificações tinha como objetivo preservar as características de reusabilidade, portabilidade e interoperabilidade dos objetos. Entretanto o foco inicial se baseou prioritariamente no conteúdo em detrimento das possíveis abordagens educacionais. Neste sentido o Learning Design, especificação da IMS, parece ser muito mais abrangente e flexível do que outros padrões existentes indo além da simples preocupação com os objetos de aprendizagem em busca da especificação de atividades de aprendizagem.

Palavras-chave: Learning Design, Objetos de Aprendizagem, Educação a Distância.

\section{Introdução}

Nos últimos anos muitas ferramentas e tecnologias têm sido utilizadas na área de Informática na Educação e Educação a Distância. Entre essas tecnologias podemos destacar os objetos de aprendizagem.

Objetos de aprendizagem podem ser definidos como qualquer material didático disponibilizado em módulos autocontidos. Atualmente existem diversas especificações que normatizam o desenvolvimento e identificação destes módulos. Entre as principais especificações podemos destacar o IMS Learning Design e o ADL SCORM (Sharable Content Objetct Reference Model).

O objetivo principal destas especificações é preservar as características de reusabilidade, portabilidade e interoperabilidade dos objetos de aprendizagem, permitindo que sejam desenvolvidos conteúdos independente de uma ferramenta de autoria, de um ambiente de aprendizagem (LMS) ${ }^{1}$ e também independente de uma plataforma de hardware e software. Partindo disso, essas especificações focaram-se principalmente na construção de metadados e no desenvolvimento de conteúdos portáveis, deixando de lado em um primeiro momento as diferentes abordagens educacionais a serem aplicadas na execução das unidades de aprendizagem. Essa lacuna em grande parte foi suprida com o surgimento da especificação EML ${ }^{2}$ (Educational Modelling Language) e posteriormente IMS Learning Design.

Este artigo visa descrever a especificação IMS Learning Design, avaliando as suas características e as ferramentas atualmente disponíveis para a utilização da mesma.

\footnotetext{
* Doutorando em Informática na Educação PPGIE/UFRGS e Mestre em Ciência da Computação PPGC/UFRGS, rdutra@pgie.ufrgs.br

** Professora Titular, Doutora em Engenharia Elétrica pela USP, Diretora do CINTED-UFRGS, liane@penta.ufrgs.br

**** Mestranda em Educação pelo PPGEDU/UFRGS e Especialista em Informática na Educação ESPIE/CINTED

${ }^{1}$ LMS (Learning Manegement System) é um ambiente de gerenciamento de aprendizagem, ou seja, são programas que envolvem ferramentas que servem para o gerenciamento e distribuição de conteúdos.

${ }^{2}$ EML (Educational Modelling Language) é uma linguagem de modelagem educacional, ou seja, um padrão para criação e administração de processos de aprendizagem. 


\section{Objetos de Aprendizagem}

Objeto de aprendizagem é qualquer recurso, suplementar ao processo de aprendizagem, que pode ser reusado para apoiar a aprendizagem, termo geralmente aplicado a materiais educacionais projetados e construídos em pequenos conjuntos visando potencializar o processo de aprendizagem onde o recurso pode ser utilizado (Tarouco, Fabre e Tamusiunas, 2003).

A idéia básica é a de que os objetos sejam como blocos com os quais será construído o contexto de aprendizagem. O projeto e criação destes objetos são realizados usando-se linguagens e ferramentas de autoria que permitem maior produtividade, uma vez que a construção dos mesmos demanda elevada quantidade de tempo e recursos, especialmente quando envolvem multimídia.

A crescente utilização de objetos de aprendizagem para apoiar atividades de ensino-aprendizagem demandou diversas iniciativas no sentido de se padronizar a especificação, construção e identificação dos mesmos.

\section{Atividades de Aprendizagem}

O conceito de objetos de aprendizagem surgiu também pela grande preocupação na padronização, desenvolvimento e na visualização de conteúdos tornado-os mais portáveis e reutilizáveis. Entretanto, essa preocupação provém em sua maioria, de cursos nos quais existem grande autonomia do aluno e aonde a principal interação existente é entre o aluno e os conteúdos, ou seja, entre o aluno e os objetos de aprendizagem. O problema é que existem cursos nos quais a ênfase da interação vai além do contato do aluno com o material educacional, querendo proporcionar também que a interação ocorra entre os próprios alunos e entre alunos e professores.

Atualmente quando se utilizam especificações como o ADL SCORM em unidades de aprendizagem que são planejadas para ter outras atividades além da visualização de objetos de aprendizagem, todas as atividades de interação do curso, ocorrem em paralelo à exploração dos objetos de aprendizagem, impossibilitando a sincronização automática entre as atividades e os objetos de aprendizagem.

Além disso, cabe salientar que existem diversas abordagens pedagógicas que podem ser utilizadas tanto em salas de aula, como em unidades de aprendizagem, tais como Projetos de Aprendizagem ${ }^{3}$, Aprendizagem Baseada em Problemas (PBL) ${ }^{4}$, entre outras. Essas abordagens são de difícil aplicação em um ambiente de aprendizagem baseando-se exclusivamente no conceito de Objetos de Aprendizagem.

\footnotetext{
${ }^{3}$ Projetos de Aprendizagem é uma abordagem pedagógica que se baseia na organização da prática pedagógica através de projetos elaborados a partir do interesse de alunos e professores e que exigem cooperação, pesquisa, curiosidade e desejo. Em seu desenvolvimento não há linearidade, pois impera o imprevisível. Essa abordagem tem como seus principais autores Hernandez e Ventura.

4 Aprendizagem baseada em problemas é uma abordagem pedagógica na qual o aprendizado é centrado no aluno. Esse modelo está baseado em problemas e na sua investigação, de forma interativa em pequenos grupos independentes, nos quais os alunos integram-se e colaboram uns com os outros. Essa abordagem tem como seus principais autores Barrows e Delisle.
} 
O conceito de Objetos de Aprendizagem, não contempla sessões de fórum, batepapo, atividades desenvolvidas pelos alunos individualmente ou em grupo, etc. Isso ocorre porque o Objeto de Aprendizagem é nada mais do que o conteúdo da unidade de aprendizagem e que nos cursos nos quais a ênfase vai além do auto-aprendizado, este pode ser considerado como somente uma parte de todo o processo que será utilizada em conjunto com outras atividades de aprendizagem (chats, discussões no fórum, diários de bordo, etc).

Neste sentido, surgiram outras iniciativas para buscar uma maior abrangência nas pesquisas referentes ao desenvolvimento de Objetos de Aprendizagem. Uma dessas iniciativas tem ganhado gradativamente destaque, principalmente por seu arcabouço conceitual, o Learning Design.

\section{Learning Design}

O Learning Design é uma linguagem de modelagem para definição de objetos e atividades de aprendizagem especificada pela IMS com base no EML (Educational Modeling Language). O EML era um sistema de notação desenvolvido pela Universidade Aberta da Holanda (OUNL), no final dos anos 90, objetivando descrever a grande variedade de modelos instrucionais existentes (Tattersal e Koper, 2003). A idéia do EML era criar uma modelagem que pudesse representar uma unidade de aprendizagem em sua totalidade, englobando não só o conteúdo como também os diversos processos envolvidos. Uma vez descrito em EML, estes modelos poderiam ser executados por um player EML, como foi realizado pela OUNL há alguns anos atrás com o software chamado Edubox (KOPER, 2002). O desenvolvimento do EML iniciouse em 1998, com base no UML (Unified Modeling Language) uma linguagem de modelagem orientada a objetos bastante utilizada na área de informática para a especificação de sistemas.

A especificação do Learning Design da IMS provê suporte ao uso de uma grande variedade de abordagens de ensino-aprendizagem, tais como: behavioristas, cognitivistas e construtivistas. Isso é possível através de uma linguagem genérica e flexível, projetada para abranger diversos tipos de abordagens pedagógicas com a mesma tecnologia. O modelo descreve "Unidades de Aprendizagem", unidades elementares que provêem eventos de aprendizagem para aprendizes, satisfazendo um ou mais objetivos de aprendizagem (TATTERSAL e KOPER, 2003).

O Learning Design (IMS, 2004) foi desenvolvido após uma extensa comparação entre as diversas abordagens pedagógicas e suas diferentes atividades de aprendizagem, para obter uma boa equalização entre generalização e aplicação pedagógica.

O Learning Design, difere de outras especificações mais voltadas ao conteúdo como o SCORM (ADL, 2004), pois parte do princípio de que no processo de ensinoaprendizagem, existem mais relações do que somente a relação de um único aprendiz diretamente com o conteúdo. A idéia é que no processo de ensino-aprendizagem, existem além desta relação citada, a relação do aprendiz com o grupo de aprendizes, com as pessoas que dão suporte e também com os recursos de aprendizagem (não somente o conteúdo, mas também as ferramentas e os objetos do mundo real).

$\mathrm{O}$ processo de ensino-aprendizagem existe quando existem atividades de aprendizagem feitas pelos aprendizes com objetivos de aprendizagem definidos. Neste sentido o Learning Design surge como um framework para a descrição deste processo 
de uma forma geral, baseado principalmente no que se convencionou uma "Unidade de Aprendizagem".

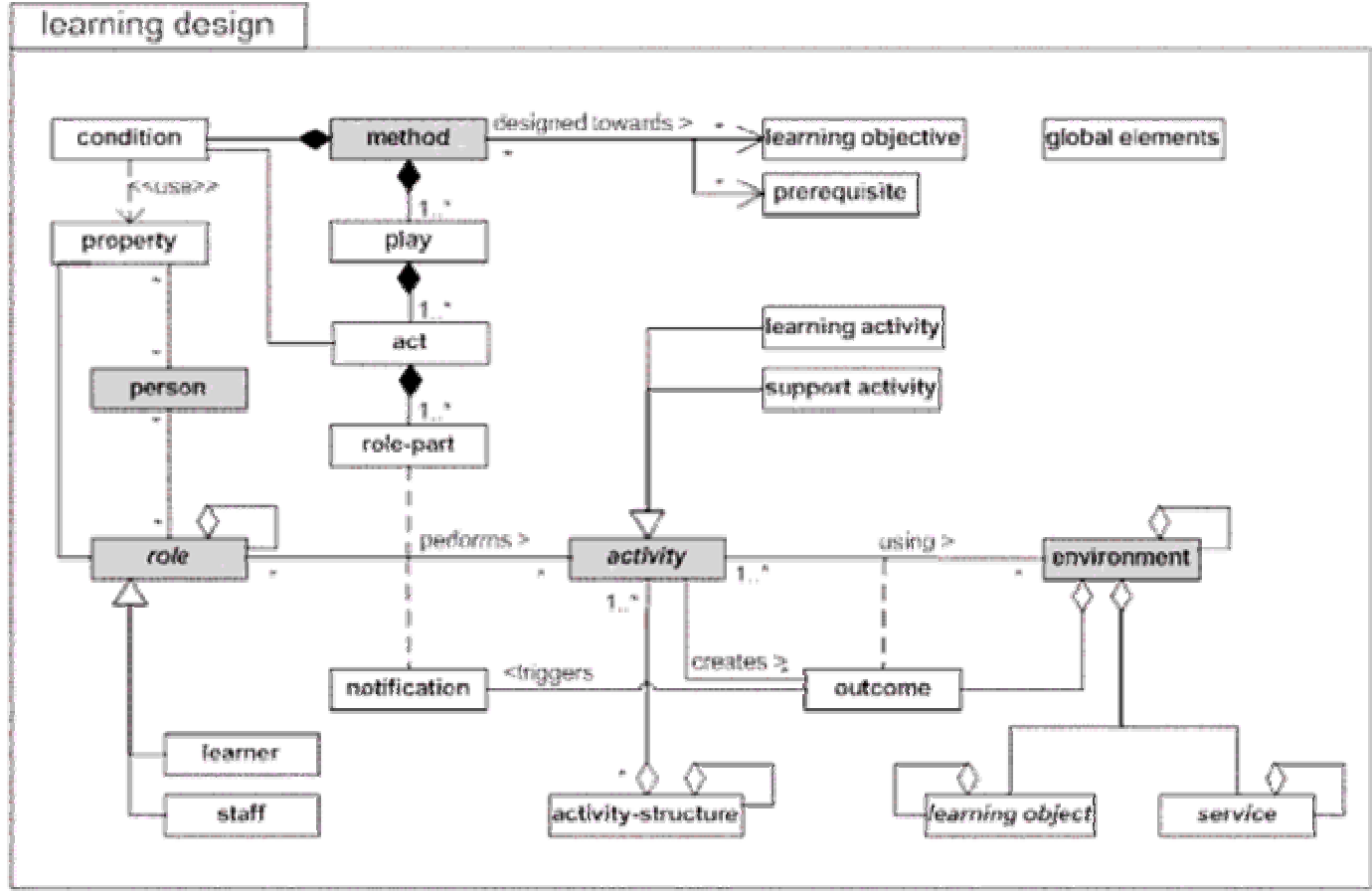

Figura 1 - Diagrama de Classes do Modelo Conceitual do Learning Design

Em uma "Unidade de aprendizagem", pessoas agem em diferentes papéis no processo de ensino-aprendizagem. Nesses papéis, elas trabalham com determinados resultados, exercendo atividades estruturadas de aprendizagem ou de suporte a aprendizagem, dentro de um ambiente. Esse ambiente consiste em objetos e serviços de aprendizagem que serão utilizados durante o encaminhamento das atividades com vistas a alcançar determinados objetivos educacionais. Nessa abordagem, ficam separados os objetos de aprendizagem e serviços do modelo educacional utilizado.

O resultado é um documento XML (Figura 2) que pode ser processado por uma aplicação "player", que coordena as interações dos estudantes e dos professores entre si e com os materiais educativos através da Web.

Uma das vantagens do LD é possuir interação com outras especificações da IMS já bastante utilizadas e difundidas, como o Content Packaging. Além disso a IMS é integrante ativa de outros órgãos de padronização e suas especificações também são utilizadas por outros padrões de objetos de aprendizagem. Outro fator, é a utilização da linguagem XML para diversos fins, desde o empacotamento das unidades de aprendizagem até a utilização de metadados.

Sua maior desvantagem era justamente a ausência de ambientes de aprendizagem e softwares de autoria que dêem suporte a esta especificação. Em sua versão original, o EML, havia a implementação do software Edubox (KOPER, 2002), que não suporta o LD, visto que apesar adoção do EML pela IMS como base para o Learning Design, as especificações são diferentes. Entretanto em 2004 a OUNL desenvolveu um Runtime Engine, chamado CopperCore, que pode servir de interface entre o ambiente de aprendizagem e as unidades LD. 


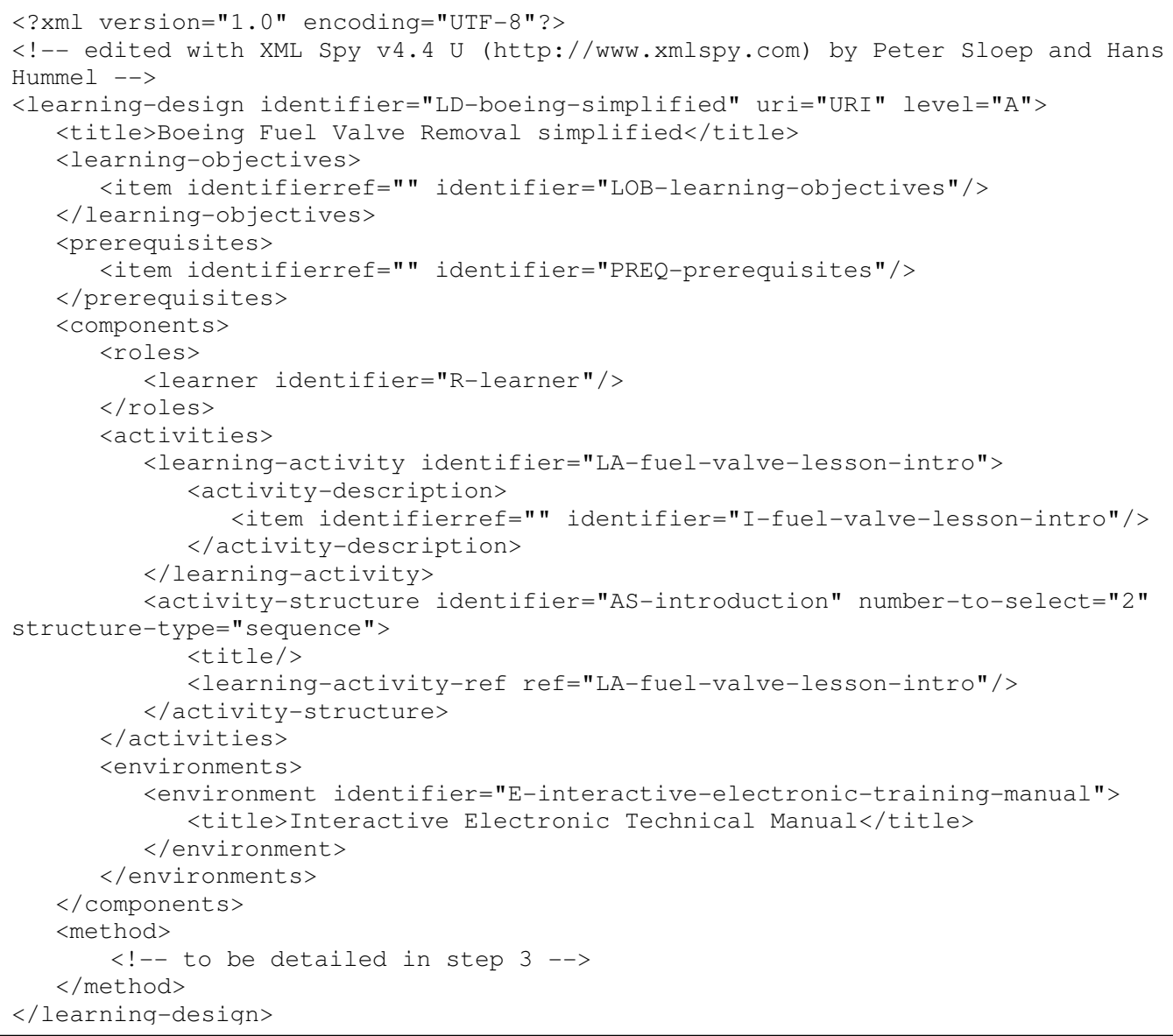

Figura 2 - Exemplo resumido de XML do Learning Design

O CopperCore é a primeira IMS Learning Design Engine de código aberto, suportando todos os três níveis do IMS Learning Design (A, B e C). Devido à complexidade exigida pelo IMS Learning Design, que contempla todas as atividades, atores e conteúdos de uma unidade de aprendizagem, o controle de todos esse processo por parte de um ambiente de aprendizagem não é uma tarefa trivial. É justamente o controle de todas estas atividades que o CopperCore fica responsável, liberando o ambiente de aprendizagem dessas tarefas.

Baseado no CopperCore, foi desenvolvido pela University of Bolton o Reload Learning Design Player. Atualmente em versão beta o LD Player permite uma fácil importação de pacotes Learning Design sem o uso de linhas de comando, automaticamente lendo e populando o LD player com um usuário ativo em cada Papel encontrado no arquivo de manifesto do IMS Learning Design.

O Learning Design Editor foi desenvolvido na University of Bolton e atualmente suporta somente o nível A. Os níveis B e C serão suportados no final de 2005. 


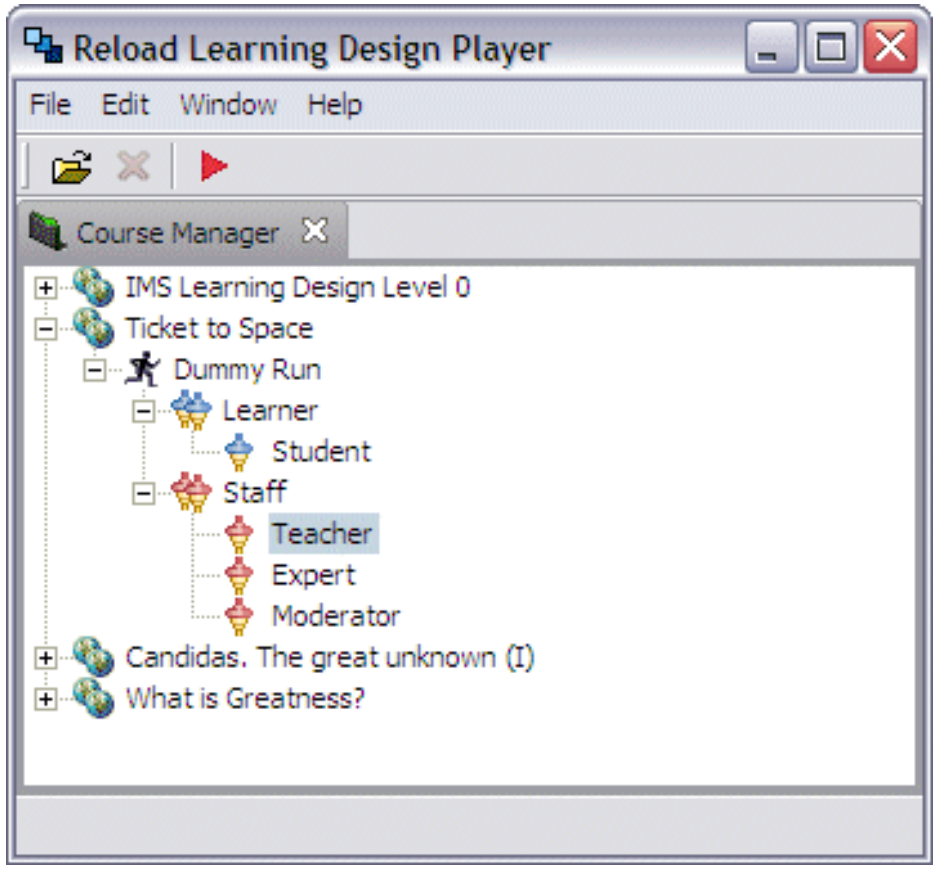

Figura 3 - Tela do Reload Learning Design Player

O LD Editor possui uma interface de usuário intuitiva e simples para a edição de projetos baseados no IMS Learning Design. Possui um gerenciador de projetos para organizar e visualizar seus projetos LD e permite a visualização e edição de arquivos dentro da ferramenta. Adicionalmente o LD Editor disponibiliza assistentes para ajudar na importação e exportação de pacotes compactados do IMS Learning Design.

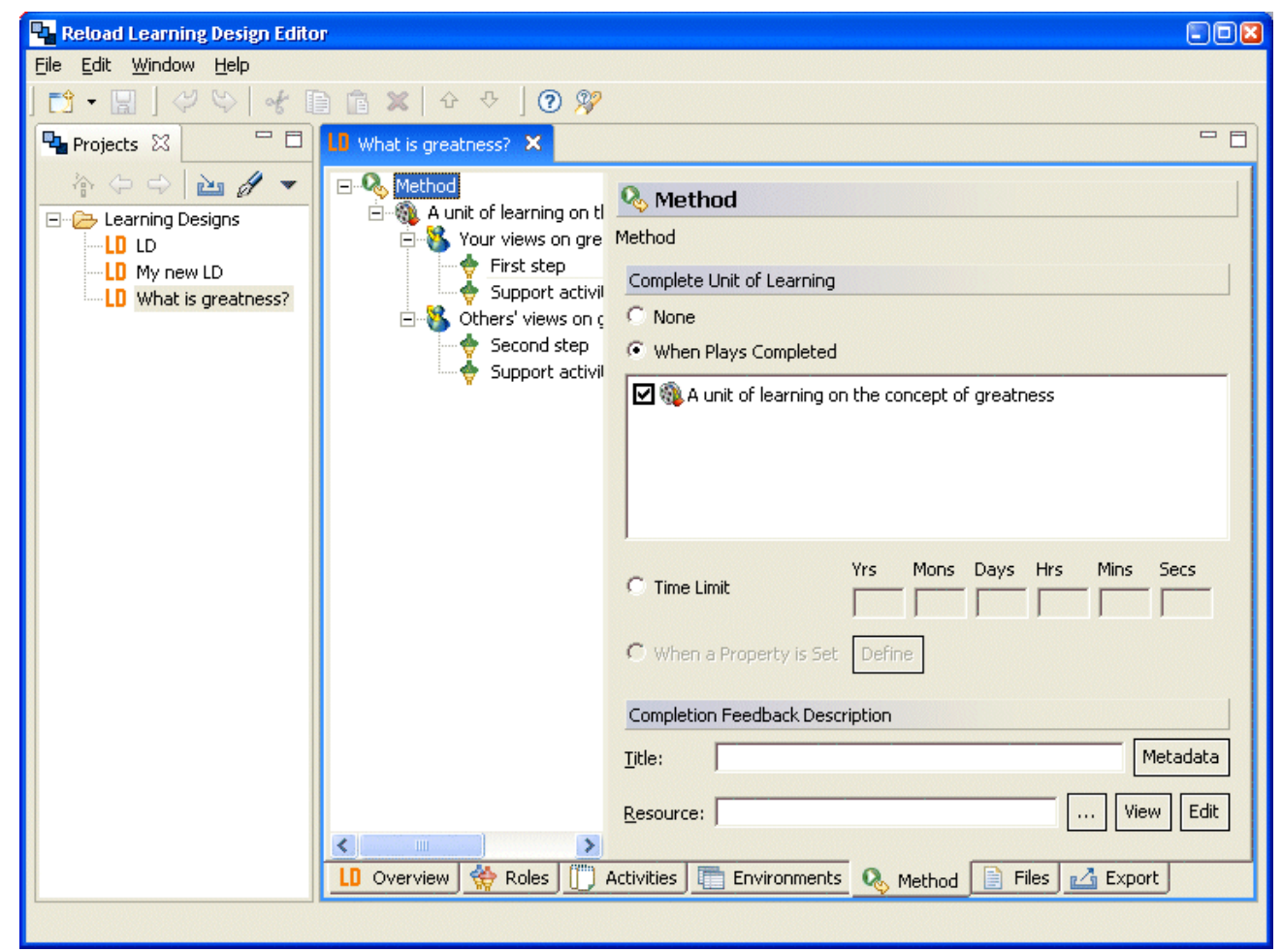

Figura 4 - Reload Learning Design Editor 
Apesar de ainda em estágio inicial essas duas aplicações em conjunto com o engine CopperCore, vêm complementar o que era considerado o grande ponto fraco desta abordagem, proporcionando aos professores e "projetistas" de unidades de aprendizagem, uma ferramenta poderosa e flexível. Mesmo assim, o IMS Learning Design ainda está longe de ter todo o suporte e aceitação de outras especificações mais consolidadas como o ADL SCORM.

\section{Conclusões}

A idéia de Objetos de Aprendizagem, inicialmente derivada do conceito de Orientação a Objetos da computação, é uma tecnologia que já provou ser bastante útil para o desenvolvimento e disponibilização de cursos e unidades de aprendizagem. Entretanto este conceito assim como na computação, se refere mais a processos repetitivos e com pouca interação além da interação entre o usuário e o sistema, entre o aluno e o conteúdo.

Assim como os processos tratados na computação, o processo de ensinoaprendizagem, vai muito além dos objetos, envolvendo atores em diferentes papéis e em diversas atividades de aprendizagem. O IMS Learning Design serve como um framework para se desenhar o processo de ensino-aprendizagem, formalizando-os em arquivos de manifesto escritos em XML. Nesse processo o professor ao invés de preocupar-se somente em como serão os Objetos de aprendizagem, irá focar-se nas Atividades de Aprendizagem e todos os recursos e atores envolvidos, nos quais estão também inseridos os Objetos de Aprendizagem.

A flexibilidade do Learning Design, permite nos desprender dos conteúdos, permitindo que adotemos com esta especificação as mais variadas abordagens pedagógicas.

\section{Referências}

Advanced Distributed Learning (ADL). Disponível em: <http://www.adlnet.org.> Acesso em: 15 jul. 2004.

BARROWS, Howard S. How to design a problem-based curriculum for the preclinical years. New York: Springer, 1985.

DELISLE, Robert. How to use Problem-Based Learning in the classroom. Alexandria: ASCD Publications, 1997.

HERNÁNDEZ, Fernando; VENTURA, Montserrat. A organização do currículo por projetos de trabalho: $O$ conhecimento é um caleidoscópio. Porto Alegre: Artes Médicas, 1998.

HERNÁNDEZ, Fernando. Transgressão e mudança na Educação. Os projetos de trabalho. Porto Alegre: Artes Médicas, 1998.

IMS - Global Learning Consortium (IMS). Disponível em: <http://www.imsproject.org/>. Acessso em 20 out. 2004. 
KOPER, R., Educational Modelling Language :adding instructional design to existingspecifications. 2002, Open University of the Netherlands.

TAROUCO, L. M. R.; FABRE M. J. M.; TAMUSIUNAS, F. R.. Reusabilidade de objetos educacionais. RENOTE - Revista Novas Tecnologias na Educação: II Ciclo de Palestras sobre Novas Tecnologias na Educação. Porto Alegre, RS, 2003 